\section{PTU-088 NON-COELIAC GLUTEN SENSITIVITY CAN BE PRESENT IN INFLAMMATORY BOWEL DISEASE, NOT JUST IRRITABLE BOWEL SYNDROME}

I Aziz*, SN Winfield, A Kelsall, N Rugg, K Pearson, J Priest, DS Sanders. Department of Gastroenterology, Royal Hallamshire Hospital, Sheffield Teaching Hospitals, Sheffield, UK

\subsection{6/gutjnl-2014-307263.162}

Introduction Self-reported gluten sensitivity (GS) commonly occurs in the absence of coeliac disease, and is termed non-coeliac gluten sensitivity (NCGS); a controversial, heterogeneous, clinical entity perceived by some to belong to the spectrum of irritable bowel syndrome (IBS) due its lack of putative biomarkers.

Aims We evaluated whether NCGS may be reported in organic gastrointestinal pathologies.

Methods A validated questionnaire screened for self-reported GS in four patient groups (cohort A); i) IBS, ii) crohns disease $(\mathrm{CrD})$, iii) ulcerative colitis (UC) and iv) gastro-oesophageal reflux disease (GORD).

In addition, the prevalence of organic pathology in a seperate group presenting and diagnosed with NCGS was also determined (cohort B).

Results Cohort A: 59 cases of IBS (mean-age 32.7 yrs, 80\% female), 75 CrD (mean-age 47.1 yrs, 59\% female), 71 UC (mean-age 43.2 yrs, 68\% female) and 109 GORD (mean-age 51.7 years, $61 \%$ female); $p$ value for age $<0.001$ and gender 0.05 .

The presence of GS was $42.4 \%$ for IBS, $29 \% \mathrm{CrD}, 25.3 \%$ UC and $18.3 \%$ for GORD. Adjusting for age and sex, IBS individuals were significantly more likely to self-report GS compared to individuals with GORD (p 0.02, OR 2.56, C. I 1.15-5.73). However, there was no difference in self-reported GS between IBS, CrD or UC.

In $\mathrm{CrD}$ the presence of strictures (p 0.04, OR 3.12, C. I 1.03-9.45) and CrD-activity index > 220 (p.0001, OR 8, C. I 2.45-2.62) were predictors of self-reporting GS. In contrast, a CrDAI score $<150$ was supportive of not being GS ( $p$ 0.002, OR 5.35, C. I 1.8-15.9). The simple colitis activity score did not influence the presence or absence of GS in UC.

Cohort B: Analysis of 200 NCGS patients (mean-age 39.1 yrs, $83 \%$ female) shows that $3 \%$ were subsequently found to have organic pathology (two cases of UC, one case each of CrD and pyloric stricture).

Conclusion NCGS is not exclusive to IBS and can also be seen in established organic gastrointestinal pathologies, such as inflammatory bowel disease. Its presence may be reflecting severe and stenotic disease. Occasionally, NCGS may be the first presentation of organic pathology.

Disclosure of Interest None Declared.

\section{PTU-089 INFLIXIMAB DOWN-REGULATES STAT 1, ALK AND P44/ 42 MAPK ACTIVATION IN CROHN'S DISEASE BIOPSIES CULTURED EX VIVO}

IM Bell*, P Giuffrida, P Biancheri, F Ammoscato, T Macdonald. CIID, Blizard Institute, Barts and The London School of Medicine and Dentistry, London, UK

\subsection{6/gutjnl-2014-307263.163}

Introduction Crohn's disease (CD) and ulcerative colitis (UC), the two main forms of inflammatory bowel disease, are characterised by increased mucosal activation of pro-inflammatory signalling molecules. The anti-tumour necrosis (TNF)-alpha monoclonal antibody infliximab is more effective in the treatment of CD than UC but its mechanism of action is still unknown. We therefore evaluated the effect of infliximab on the expression of a panel of phospho-proteins by inflamed CD and UC colonic biopsies cultured ex vivo.

Methods Colonic biopsies were obtained from macroscopically inflamed areas of 5 patients with CD and 2 patients with UC, and were then cultured for $24 \mathrm{~h}$ in $300 \mathrm{ul}$ of serum-free HL-1 medium with infliximab $(5 \mathrm{ug} / \mathrm{ml})$, or control $\mathrm{IgG} 1(5 \mathrm{ug} / \mathrm{ml})$. The biopsies were then snap frozen and later lysed to extract the protein. A Path Scan RTK signalling array kit from New England Biolabs was used to measure the expression of a panel of 39 phosphorylated proteins in the biopsy homogenates.

Results Infliximab significantly reduced the expression of phosphorylated ALK, FLT3, EphB3,p44/42 MAPK, S6 Ribosomal Protein and Stat 1 by over 40 fold compared to IgG1 in Crohn's disease biopsies cultured ex vivo. In UC biopsies infliximab did not induce any significant change in phosphoprotein expression compared to IgG1 control except for a 10 fold reduction in phospho-VEGFR2.

Conclusion Infliximab reduces the expression phospho-proteins Stat 1 , ALK and p44/42 MAPK, which have a central role in sustaining the pro-inflammatory immune response. Differences in the effect of infliximab on the phosphorylation status of mucosal proteins may account for its different efficacy profile in $\mathrm{CD}$ and UC.

Disclosure of Interest None Declared.

\section{PTU-090 10 YEAR RETROSPECTIVE REVIEW OF ABDOMINAL TUBERCULOSIS FROM A LONDON TEACHING HOSPITAL: AGE, ETHNICITY AND HIV STATUS}

${ }^{1}$ JS Nayagam*, ${ }^{2} \mathrm{C}$ Mullender, ${ }^{1} \mathrm{~A}$ Poullis, ${ }^{2} \mathrm{C}$ Cosgrove. ${ }^{1}$ Gastroenterology and Hepatology, St George's Hospital, London, UK; ${ }^{2}$ Clinical Infection Unit, St George's Hospital, London, UK

\subsection{6/gutjnl-2014-307263.164}

Introduction Although tuberculosis (TB) rates in United Kingdom have plateaued in recent years, areas with large high risk non-UK born population, which are predominantly centred in large urban areas, still have a high prevalence of TB. Extra-pulmonary $\mathrm{TB}$, including abdominal $\mathrm{TB}$, is much more common in non-UK born than in UK born population. The highest rate of $\mathrm{TB}$ in UK born is in the over 75 years of age population. Abdominal TB can mimic Crohn's disease and should always be considered as part of the differential diagnosis. We sought to review the demographics of patients with abdominal tuberculosis in a large urban teaching hospital in London.

Methods A retrospective review of patients treated at $\mathrm{St}$ George's Hospital, London, for abdominal TB from June 2003 to August 2013 was conducted. Information was gained from electronic patient records and the hospital's tuberculosis database.

\begin{tabular}{lll} 
Abstract PTU-090 Table 1 & & \\
\hline & Number (\%) & Average age \\
\hline South Asian & $34(52.3 \%)$ & 42.3 \\
African & $25(38.5 \%)$ & 36.1 \\
European Caucasian & $5(7.7 \%)$ & 72.4 \\
Caribbean & $1(1.5 \%)$ & 28 \\
\hline
\end{tabular}

